\title{
State of Art
}

\section{Study on challenges in diagnosis of TB and MDR TB by Gene-Xpert in Bangladesh}

\author{
Haq Rouseli, Bichha R.P., Uddin Md. Ashraf, Modak Pronab Kumar, Rahman Md. Mojibur
}

\begin{abstract}
TB is a leading cause of morbidity and mortality worldwide. However, public health services globally reported only $66 \%$ of the estimated TB cases in 2014. Moreover, less than $5 \%$ of notified TB cases were tested for drug resistance which is often diagnosed after prolonged diagnostic delays. The main reasons for these gaps are inadequate diagnostic capacity and an over reliance on chest radiography and/or sputum smear microscopy as diagnostic tools. The "classical" diagnosis of HIV- associated and drug-resistant TB is complex, expensive, slow and technically demanding, relying on conventional culture and drug susceptibility testing (DST). Detecting more cases, detecting them early and rapidly identifying drug resistance are essential for improving individual patient health and avoiding transmission in the community. The Xpert MTB/RIF assay is an automated, real-time nucleic acid amplification technology represents a paradigm shift in the diagnosis of TB and drug-resistant TB by simultaneously detecting Mycobacterium tuberculosis and Rifampicin resistance-conferring mutations in a closed system suitable for use outside conventional laboratory settings in less than 2 hours, directly from sputum samples.

Objective: To find out the challenges in diagnosis of TB and MDR TB by Gene-Xpert in Bangladesh

Methodology; Both quantitative and qualitative methods study designs were used. All the 43 Centres in Bangladesh where the Gene $X$ pert test are carried out were included. In addition selective officials/staff from national and sub-national level were included as respondents for focus group discussion (FGD) and in-depth interview. Verbal informed consent was obtained from participants before starting interviews. Analysis of data was done using SPSS software program.
\end{abstract}

Results: Xpert MTB/RIF was first introduced in Bangladesh in March 2012. Till December 2015, a total of 61 Xpert MTB/ RIF machines were functioning at 43 sites in the country. In 42 sites the GeneXpert machines are placed in a separate room and in one site it is placed along with other general pathological laboratory activities. It was found that in all sites necessary physical support like air-cooler, dust control, UPS as well as regular electricity and water supply were available. Out of 43 sites, 38 have 4-module and 5 sites have 16-module machine. In totall, 55 four module machines and 6 sixteen module have been established. Out of these only one 4modul machine was non-functioning. In about half (48.8\%) of the sites machines are run twice a day and in 17 sites (39.5\%) only once while in 5 sites (NGO supported sites) machines are run 3 times a day. In 19 (44.2\%) sites 1-4 samples are tested per day and in 16(37.2\%) sites 5-8 samples/day. Per day 9-20 samples are tested in 4(9.3\%) centres while in other 4 centres 21-30 samples are tested daily. In all the centres a total of about 300 samples are tested in a single working day. In total, 38 sites informed about problems they faced in operating the machine. The most common problem was module failure (67.4\%), followed by delay in maintenance support (46. 5\%). Inadequate cartridge supply and load shading were faced by $16.3 \%$ and $11.6 \%$ respectively. In 42 sites there were needs for support. The most common support they need is refresher training (93\%), followed by maintenance training by $79.1 \%$, and Software training by $18.6 \%$. The responses from both FDG and in-depth interviews were as follows:

Most common problem faced by the heath workers were lack of timely maintenance of Machines, false result of Rifampacin Resistance due to low bacterial load, module failure, no proper sputum transport mechanism, lack of appropriate centrifuge machine for processing of samples of EP cases and inadequate man power. From the past experience the group provided some valuable suggestions and comments as follows;

A well maintained sputum transportation mechanism to be established. Stable power supply is absolutely necessary as discontinuation of electricity even for a fraction of second will cause erroneous test result. More machines need to made available for easier access. Machine operator needs refresher training including training related to day- today maintenance and software system. Good quality sample in sufficient amount to be ensured for Xpert testing to produce accurate results.

Conclusion: Gene-Xpert machine is very useful in diagnosis of MDR and Rifampacin Resistance M. tuberculosis. Module failure is a common problem and their replacement takes longer time. Frequent errors are shown that might be due to poor quality of sample, unstable electricity supply or poor skill of machine operators. Proper training of operators and proper sputum transport system is urgently needed for efficient use of these machines.

\section{Correspondence:}

Dr. Md. Mojibur Rahman,

National Progream Co-ordinator, NTP, DGHS, Bangladesh,

Email:drmdmojib@gmail.com 


\section{Introduction:}

Tuberculosis (TB) is a major global health problem. It causes ill-health among millions of people each year and ranks alongside the human immunodeficiency virus (HIV) as a leading cause of death worldwide. Bangladesh is among 30 countries with the high burden of TB and MDR-TB. The estimated incidence rate of all forms of tuberculosis was 225 per 100 , 000 population in 2015. In 2015 a total of 206,915 new and relapse cases were notified, among them $4 \%$ cases aged less than 15 years. The overall male female ratio was 1.5 in 2015. The treatment success rate among new and relapse cases (all forms) is above 90\% since 2007. In 2014 cohort the overall treatment success rate was $93 \%$ and among bacteriologically confirmed cases it was 94\%. (1) NTP Bangladesh has conducted countries first nationwide drug resistance survey in 20102011. According to this survey report the proportion of new TB cases with MDR-TB is $1.4 \%$ and that of retreatment cases with MDR-TB is $28.5 \%$. On this assumption the estimated total number of MDR-TB cases in 2015 in the country is 4662 (2198 among new PTB cases and 2464 among retreated PTB cases). ${ }^{(1)}$

People with MDR-TB or RR-TB are eligible for second-line treatment with MDR-TB regimens. Globally, a total of 111000 people were started on MDR-TB treatment in 2014, an increase of 14\% compared with 2013. Only 50\% of patients on MDRTB treatment were successfully treated, largely due to high rates of mortality and loss to follow-up ${ }^{(2-4)}$

The SAARC region, with an estimated annual incidence of 3.1 million TB cases, carries $32 \%$ of the global burden of TB incidence. Three of the eight Member Countries in the Region are among the 30 TB and MDR-TB high burden countries, with India accounting for $23 \%$ of the world's TB cases.

In 2014, there were an estimated 3.1 million incident cases of TB, equivalent to 185 cases per 100000 population in the region. This carries $31 \%$ of the global burden of TB incidence. The absolute number of incident cases is falling slowly, from 2000 to 2014. (2)

The MDR TB cases in the region range from less than one to four percent (1-4\%) among new TB cases and it ranges from less than one to almost 35 percent among the retreatment TB cases.

\section{MDR-TB situation in Bangladesh (3)}

The results of the first national DRS completed in 2012 confirmed a low proportion of new TB cases that have MDR-TB $(1.4 \%$, confidence intervals $0.7-$ $2.5)$, but the proportion among retreated cases was revised upwards $(28.5 \%$, confidence intervals $24-$ 34). The total number of estimated MDR-TB cases among notified cases in 2015 was 4662. Coverage of routine surveillance of drug resistance is still low. ${ }^{(3)}$

\section{Issues related to conventional method of diagnosis TB:}

With 9.6 million incident cases of TB and 1.5 million deaths estimated in 2014 TB is a leading cause of morbidity and mortality worldwide. However, public health services globally reported only $66 \%$ of the estimated TB cases in 2014. Moreover, less than 5\% of notified TB cases were tested for drug resistance which is often diagnosed after prolonged diagnostic delays ${ }^{(4-6)}$

The main reasons for these gaps are inadequate diagnostic capacity and an over reliance on chest radiography and/or sputum smear microscopy as diagnostic tools. Patients with HIV-associated TB, those with sputum smear-negative and/or extra pulmonary disease, and drug-resistant TB patients are particularly affected by the failure of microscopy as a primary diagnostic tool. The "classical" diagnosis of HIV- associated and drug-resistant TB is complex, expensive, slow and technically demanding, relying on conventional culture and drug susceptibility testing (DST). The long delay (up to several weeks) required to obtain results has devastating consequences for patients who go undiagnosed (and therefore untreated or inappropriately treated), or are diagnosed too late ${ }^{(7)}$. Detecting more cases, detecting them early and rapidly identifying drug resistance are essential for improving individual patient health and avoiding transmission in the community. This requires universal access and early detection using contemporary tools and innovative strategies $(8,9,10)$

The past decade has seen unprecedented growth in the TB diagnostic pipeline and accelerated efforts to establish the necessary laboratory infrastructure ${ }^{(10)}$. Nevertheless, although recommended by WHO, the latest generation liquid culture diagnostics and molecular line probe assays for rapid detection of MDR-TB have not yet solved the diagnostic dilemma in most resource-limited settings, largely due to the need for expensive laboratory infrastructure, extensive bio-safety precautions and specialized staff. ${ }^{(10)}$

A new rapid test that overcomes many of the current operational difficulties was recommended for use by WHO in December 2010: the Xpert MTB/RIF assay (Cepheid, Sunnyvale, CA, USA) is an automated, real-time nucleic acid amplification technology run on the multi-disease platform GeneXpert (Cepheid). The Xpert MTB/RIF assay represents a paradigm 
shift in the diagnosis of TB and drug-resistant TB by simultaneously detecting Mycobacterium tuberculosis and Rifampicin resistance-conferring mutations in a closed system suitable for use outside conventional laboratory settings in less than 2 hours, directly from sputum samples ${ }^{(11,12)}$

\section{Field demonstration studies revealed that,}

Xpert MTB/RIF detected $90.3 \%$ of the cultureconfirmed TB cases, compared with $67.1 \%$ using microscopy. In sputum smear- negative, culturepositive TB patients Xpert MTB/RIF test sensitivity was $76.9 \%$ and specificity was $99.0 \%$. Sensitivity for rifampicin resistance was $94.4 \%$ and specificity was $98.3 \%{ }^{(13)}$

While same study revealed that, HIV co-infection substantially decreased the sensitivity of microscopy (to 47\%), Xpert MTB/RIF performance was not significantly affected. The median time to detection of TB was 0 days (inter-quartile range (IQR) $0-1$ ) using Xpert MTB/RIF, compared to 1 day (IQR 0-1) for microscopy, 30 days (IQR 23-43) for solid culture and 16 days (IQR 13-21) for liquid culture. The median time to detection of rifampicin resistance was 20 days (IQR 10-26) for line-probe assay versus 106 days (IQR 30-124) for phenotypic DST.(13)

The Xpert MTB/RIF test reduced the median time to treatment for sputum smear-negative TB from 56 days (IQR 39-81) to 5 days (IQR 2-8). The indeterminate rate of Xpert MTB/RIF testing was $2.4 \%$ (126 out of 5321 samples) compared to $4.6 \%$ (441 out of 9690 ) for culture. ${ }^{(13)}$

Previous studies of the MTB/RIF assay have reported test sensitivities of 57 to $76.9 \%$ in cases of smear-negative, culture-positive pulmonary tuberculosis and 98 to $100 \%$ in cases of smearpositive, culture-positive pulmonary tuberculosis, while the test specificity remained at $99 \%$ to $100 \%$ $(17,18,19,20$, $)$

In the previous studies, the sensitivity of the MTB/ RIF test for detecting RIF resistance was 94.4 to $100 \%$ and the specificity was 98.3 to $100 \%{ }^{(14)}$.

\section{Operational and logistical issues:}

The available evidence confirmed the robustness of the Xpert MTB/RIF assay under varying temperature and humidity conditions, the need for minimal staff training, basic bio-safety requirements (as for sputum smear microscopy) and high levels of user satisfaction. Operational challenges included the requirement for an ambient temperature $<30^{\circ} \mathrm{C}$ (necessitating air conditioning in hot climates), and uninterrupted and stable electrical power supply (requiring generators in several sites). Storage space and conditions $\left(28^{\circ} \mathrm{C}\right.$ for cartridges, waste generated (considerably more than for microscopy), and the 12-month shelf-life of cartridges were listed as main operational challenges $(15,16)$ Cost, affordability and cost-effectiveness analyses Using Xpert MTB/RIF for the diagnosis of smear-negative pulmonary TB was deemed cost-effective compared with existing diagnostic strategies in India, South Africa and Uganda, and within WHO acceptable incremental cost effectiveness ratios $(14,15,16)$

The Gene-Xpert MTB/RIF assay is a novel integrated diagnostic device that performs sample processing and heminested real-time PCR analysis in a single hands-free step for the diagnosis of tuberculosis and rapid detection of RIF resistance in clinical specimens. The assay can generally be completed in less than 2 hours ${ }^{(17)}$.

Xpert MTB/RIF was first introduced in Bangladesh in March 2012 with the USAID supported TB CARE II project. Till December 2015, Xpert MTB/ RIF machines were functioning at 43 settings (including 5 sites supported by icddr,b) in the country. Since inception of this method, no evaluation the challenges in diagnosis of TB and drug resistant TB in Bangladesh was done in a systematic way. Hence according to the decision of the $25^{\text {th }}$ Governing Board Meeting of the STAC this study was conducted in Bangladesh form 06 January to 05 April 2017 to achieve the following objective.

Objective: To find out the challenges in diagnosis of TB and MDR TB by Gene Xpert in Bangladesh

\section{Methodology;}

Study settings: All the institutions in Bangladesh where the Gene $X$ pert test ( 43 Centres in Bangladesh) are carried out were included in the study. In addition selective officials/staff from national and sub-national level were included as respondents for focus group discussion (FGD) and in-depth interview.

\section{Study Design:}

This study used both qualitative and quantitative research methods and accordingly research instruments were developed to address the research objectives.

In quantitative part information related to Xpert Machines and Machine operator were included. In qualitative section Focus Group Discussion (FGD) and Key informant in-depth interviews were done.

Three FGDs were carried out with i) National level officials related to TB control and are involved in GeneXpert procurement, establishment, functioning 
and supervision/ monitoring ii)Microbiologists/ Laboratory Technicians who are involved in Gene X- Pert Procedure

iii) Medical Officers/health workers who are currently treating/managing MDR TB patients. A set of standard questionnaire was used for conducting FDG.

In addition to FDG few key Informant Interviews were carried out with the key personnel involved in Gene X Pert technique in national and sub-national level of the country.

Verbal informed consent was obtained from participants before starting interviews.

Analysis of quantitative data was done using SPSS software program. All the information from interview and FGDs were checked for ensuring quality of the data. Data coding/programming was done for entering the data. Then data were entered, and checked/ cleaned. Data analysis was done through frequency table and cross table on the basis of identified variables, and accordingly results/findings were highlighted in the draft report which was finalized after review and dissemination.

\section{Findings}

Xpert MTB/RIF was first introduced in Bangladesh in March 2012. Till December 2015, a total of 61 Xpert MTB/ RIF machines were functioning at 43 sites in the country. Data have been collected to achieve the objectives from all these 43 sites. During 2016 no machine was installed.

Seventeen (39.5\%) out of total 43 GeneXpert sites are in Dhaka division and it includes five sites supported by ICDDR,B which working for TB control as a partner of NTP (Table 1). In 42 sites the GeneXpert machines are placed in a separate room and in one site it is placed along with other general pathological laboratory activities. During data collection it was found that in all sites necessary physical support like air-cooler, dust control (inbuilt with Xpert machine), UPS as well as regular electricity and water supply were available. However in 2 sites air cooler was found non-functioning and in other 2 sites UPS was non-functioning (Table 2) which were under process of repair.

A total of 65 operators are operating the machines in 43 Gene Expert sites. In 23 centres only one operator, in 18 centres there are 2 operators and in 2 centres (Rajshahi CDH and Chittagong RTRL) 3 operators are working considering the workload (Table 3).

During data collection where 2 or 3 operators were involved, interview was taken only from the senior one. So in total interview were taken from 43 operators. Among those 35 ( $81.4 \%$ ) were male and
8 ( 18.6\%) were female. Their age ranged from 23 to 55 years with a mean age $32.28 \pm 8$ years. More than $50 \%$ were less than 30 years, 14 were within the range of 30-44 years and only 7 were of more than 44 years, and these all 7 were males (Table 4).

\section{Other key parameters/characteristics of the Machine operators:}

Regarding general education, more than one- third of the operators were only SSC pass and less than onefourth of them were HSC pass. About $87 \%$ (36/43) of them have lab related formal institutional educational background (e.g. Diploma in Medical Technology Lab) and seven has no such background or even no formal lab related training. Professional designations are not harmonized among government and nongovernment organizations. More than 50\% (22/43) has professional designation as MT Lab (under GOB) , while $14(32.6 \%)$ are designated as Technical Asst. Lab (under NGO). Other designations are health worker (4), senior lab technician, research assistant and lab attendant (each one). All MT labs are qualified with Diploma in MT Lab and are Supported by Government of Bangladesh. On the other hand most of the operators supported by NGO (BRAC and ICDDR,B) are not diploma holder.

Length of Service: More than $60 \%$ (26) have been working for 30-40 months, $14 \%$ ( 6) for 50-59 months, 3 for 2 months and 8 (18.6\%) for less than I year. All except two(2) have received 3 day training on GeneXpert machines ( 36 from form NTP and 5 from ICDDR,B). The other 2 have working experience with trained colleagues and will also receive formal training. (Table 5). Length of service varies because of phase wise installation of machines and training also provided phase wise by NTP with the support of NTP and Challenge TB USAID.

\section{Type and number of machines:}

Out of 43 sites, 38 have 4-module and 5 sites have 16-module machine. In total 55 four module machines ( 34 under NTP and 21 under ICDDR,B) and 6 sixteen module machines (all under NTP) have been established. Out of these only one 4modul machine was non-functioning (for five days due to software problem). Therefore total number of module is 316 (Table 6-9)

The table 10 shows that in 23 sites there were non functioning modules and out of total 316 modules 57 (18.04\%) were non-functioning. Higher proportion of nonfunctioning modules might be due to lengthy process of module replacement by Cepheid, Netherland.

On an average in about half (48.8\%) of the sites machines are run twice a day, in 17 sites (39.5\%) 
only once while in 5 sites (NGO supported sites) machines are run 3 times a day (Table 11).

In 19 (44.2\%) sites 1-4 samples are tested per day and in $16(37.2 \%)$ sites $5-8$ samples/day. Per day 9-20 samples are tested in 4(9.3\%) centres while in other 4 centres 21-30 samples are tested daily. In all the centres a total of about 300 samples are tested in a single working day (Table 12).

In total, 38 sites informed about problems they faced in operating the machine. The most common problem was module failure $(67.4 \%)$, followed by delay in maintenance support (46.5\%). Inadequate cartridge supply and load shading were faced by $16.3 \%$ and $11.6 \%$ respectively (Table 13 ).

In 42 sites there were needs for support. The most common support they need is refresher training (93\%), followed by maintenance training by $79.1 \%$, and Software training by $18.6 \%$. Only $3(7.3 \%)$ centres asked for more Xpert machine. (Table 14).

In-depth interview was taken from 23 respondents form different institutions/organization; among them 11 were from Government organization and 12 from NGO; their distribution is shown below in Table 15 and 16:

\section{For in-depth interview 3 questions were set as below:}

- Problem faced in running the GeneXpert machine in diagnosis of TB and Gene Expert

- Any lesson Learned: though using the machines in diagnosing TB cases:

- Suggestions (if any) for further improvement. We also arranged 4 Focus Group Discussions for officials of different levels to identify the challenges and probable suggestions for further improvement in optimum utilization of GeneXpert machines in diagnosis of TB and MDR TB.
The responses from both FGD and in-depth interviews were analyzed and were summarized in 3 broad areas as shown in Table 17.

\section{Conclusion:}

- Availability of GeneXpert Machine are inadequate in comparison to need.

- Though almost $100 \%$ of the available machines are functional, there is lack of adequate support for timely maintenance.

- Module failure is a common problem and their replacement takes longer time. (>18\% of total modules are found nonfunctional).

- Frequent errors are shown that might be due to poor quality of sample, unstable electricity supply or poor skill of machine operators. (only 3 days training received). Also might be due non existence of well established sputum transport mechanism.

- Non-existence of EQA

- Strong monitoring and supportive supervision is key to improve the situation.

\section{Recommendation:}

- Rapid expansion of GeneXpert facility is required to enhance detection of TB and MDR TB

- Diagnostic algorithm needs to be revised to testing all presumptive TB and DR TB

- System to be developed for timely maintenance of machines

- $\quad$ Refresher training for Machine operator needs to be arranged

- To ensure quality EQA system to be established.

- Supervision/monitoring to be strengthened.

Table 1. Distribution of GeneXpert Sites by Division $(\mathrm{N}=43)$

\begin{tabular}{|l|l|c|c|c|}
\hline SI No. & \multicolumn{1}{|c|}{ Division } & Frequency & Percent & Remarks \\
\hline 1 & Rajshahi & 3 & 7.0 & \\
\hline 2 & Rangpur & 4 & 9.3 & \\
\hline 3 & Khulna & 5 & 11.6 & \\
\hline 4 & Barisal & 3 & 7.0 & \\
\hline 5 & Dhaka & 12 & 27.9 & \\
\hline 6 & Sylhet & 3 & 7.0 & \\
\hline 7 & Chittagong & 8 & 18.6 & \\
\hline 8 & ICDDR,B & 5 & 11.6 & All these 5 sites are in Dhaka \\
\hline & Total & 43 & 100.0 & \\
\hline
\end{tabular}


Table 2 GeneXpert Room status and required facilities ( $N=43$ )

\begin{tabular}{|l|l|l|l|}
\hline Status/facilities & \multicolumn{1}{|c|}{ Yes (\%) } & \multicolumn{1}{|c|}{ No (\%) } & \multicolumn{1}{c|}{ Remarks } \\
\hline $\begin{array}{l}\text { Placed in } \\
\text { separate room }\end{array}$ & $42(97.7)$ & $1(2.3)$ & Placed along with general Lab activities \\
\hline Air cooler & $43(100)$ & 00 & 2 were non functioning \\
\hline Dust control & $43(100)$ & 00 & Inbuilt with Xpert machine \\
\hline UPS & $43(100)$ & 00 & 2 were non functioning \\
\hline Regular Electricity & $43(100)$ & 00 & \\
\hline Running Water supply & $43(100)$ & 00 & \\
\hline
\end{tabular}

Table 3. Number of operator per site $(\mathrm{N}=43)$

\begin{tabular}{|c|c|c|c|}
\hline Number of operator & Frequency (Number of Sites) & Percent & Remarks \\
\hline 1 & 23 & 53.5 & \\
\hline 2 & 18 & 41.9 & \\
\hline 3 & 2 & 4.7 & Considering higher workload \\
\hline Total & 43 & 100.0 & \\
\hline
\end{tabular}

Table 4. Age distribution of the machine operators $(\mathrm{N}=43)$

\begin{tabular}{|c|c|c|c|}
\hline \multirow{2}{*}{ Age group (yrs) } & \multicolumn{2}{|c|}{ Frequency } & \multirow{2}{*}{ Total (\%) } \\
\cline { 2 - 3 } & Male (\%) & Female (\%) & \\
\hline $23-29$ & $17(77.3)$ & $5(22.2)$ & $22(51.2)$ \\
\hline $30-44$ & $11(78.6)$ & $3(21.4)$ & $14(32.5)$ \\
\hline $45-55$ & 7 & 0 & $7(16.3)$ \\
\hline Total & $35(81.4)$ & $8(18.6)$ & $43(100)$ \\
\hline
\end{tabular}

Table 5. Some other key parameters of the machine operators $((\mathrm{N}=43)$

\begin{tabular}{|c|c|c|c|}
\hline parameters & Frequency & $\%$ & Remarks \\
\hline \multicolumn{4}{|l|}{ Education (general): } \\
\hline SSC & 29 & 67.4 & \\
\hline $\mathrm{HSC}$ & 10 & 23.3 & \\
\hline Bachelor & 3 & 7.0 & \\
\hline Master & 1 & 2.3 & \\
\hline Total & 43 & $100 \%$ & \\
\hline \multicolumn{4}{|l|}{ Education (technical): } \\
\hline None & 7 & 16.3 & \\
\hline Laboratory related & 36 & 83.7 & \\
\hline Total & 43 & $100 \%$ & \\
\hline \multicolumn{4}{|l|}{ Professional Designation } \\
\hline MT Lab & 22 & 51.2 & \\
\hline Technical Asst. Lab & 14 & 32.6 & \\
\hline Senior Lab Technician & 1 & 2.3 & \\
\hline Lab attendant & 1 & 2.3 & \\
\hline Research Assistant & 1 & 2.3 & \\
\hline
\end{tabular}




\begin{tabular}{|c|l|l|l|}
\hline Health worker & 4 & 9.3 & \\
\hline Total & 43 & $100 \%$ & \\
\hline Working Duration & \multicolumn{5}{|l|}{} \\
\hline <1 year (2-23 months) & 8 & 18.6 & \\
\hline 1-2 year (24 months) & 3 & 7.0 & \\
\hline $30-48$ months & 26 & 60.4 & \\
\hline$>4$ years (50-59 months) & 6 & 14.0 & \\
\hline Total & 43 & $100 \%$ & \\
\hline Training Received on GeneXpert & 41 & 95.3 & \\
\hline Yes & 2 & 4.7 & \\
\hline No & 43 & $100 \%$ & \\
\hline Total & 36 & 83.7 & \multirow{2}{*}{ All received 3 days training } \\
\hline Training Received from & 5 & 11.6 & On the Job training form Trained \\
\hline NTP (NTRL) & 2 & 4.7 & operator who has left the job \\
\hline ICDDR,B & 2 &
\end{tabular}

Table 6. Type of machines based on number of modules $(\mathrm{N}=43)$

\begin{tabular}{|l|l|l|l|}
\hline Machine Type & Frequency & \multicolumn{1}{|c|}{$\%$} & \multicolumn{1}{|c|}{ Remarks } \\
\hline 16 Model machine & 5 & 11.6 & Under ICDDRB, \\
\hline 4 module Machine & 38 & 88.4 & \\
\hline Total & 43 & $100 \%$ & $\begin{array}{l}\text { there are four 4modules in 2 centres and five } \\
\text { tmodules in 1 centre }\end{array}$ \\
\hline
\end{tabular}

Table 7. Type and number of Machines per site $(\mathrm{N}=43)$

\begin{tabular}{|c|c|c|c|c|}
\hline Type & Number & Frequency & Percent & Remarks \\
\hline \multirow{6}{*}{4 modules } & 0 & 5 & 11.6 & \\
\hline & 1 & 34 & 79.1 & 34 machines under NTP \\
\hline & 4 & 2 & 4.7 & \multirow{3}{*}{21 machines under ICDDR,B } \\
\hline & 5 & 1 & 2.3 & \\
\hline & 8 & 1 & 2.3 & \\
\hline & Total & 43 & 100.0 & \\
\hline \multirow{4}{*}{16 module } & 0 & 38 & & \\
\hline & 1 & 4 & & \multirow{2}{*}{ All 6 machines are under NTP } \\
\hline & 2 & 1 & & \\
\hline & Total & 43 & 100.0 & \\
\hline
\end{tabular}

Table 8. Summary table for calculating number of machine and number of modules

\begin{tabular}{|l|l|l|l|}
\hline Machine Type & \#of sites where placed & Total machine & Number of modules \\
\hline 4- module & 38 & 55 & 220 \\
\hline 16- module & 5 & 6 & 96 \\
\hline Total & 43 sites & 61 machines & 316 modules \\
\hline
\end{tabular}


Table 9 Functional status of Machines

\begin{tabular}{|l|c|c|c|}
\hline \multicolumn{1}{|c|}{ Machine Type } & Functioning & nonfunctioning & total \\
\hline 4- module & 54 & 1 & 55 \\
\hline 16- module & 6 & 0 & 6 \\
\hline Total & 60 & 1 & 61 \\
\hline
\end{tabular}

Table 10 Functional status of modules by sites

\begin{tabular}{|l|l|l|l|l|}
\hline $\begin{array}{c}\text { Number of non } \\
\text { functioning } \\
\text { modules } \\
\text { (i) }\end{array}$ & $\begin{array}{c}\text { frequency } \\
\text { (sites) } \\
\text { (ii) }\end{array}$ & \% & $\begin{array}{c}\text { Total non } \\
\text { functioning } \\
\text { modules } \\
\text { (i)x(ii) }\end{array}$ & Remarks \\
\hline 0 & 20 & 46.5 & 00 & \\
\cline { 1 - 4 } & 9 & 20.9 & 9 & \multirow{2}{*}{ Total nonfunctioning modules } \\
\hline 2 & 8 & 18.6 & 16 & \\
\hline 4 & 4 & 9.3 & 16 & \\
\hline 7 & 1 & 2.3 & 7 & \\
\hline 9 & 1 & 2.3 & 9 & \\
\hline Total & 43 & 100.00 & 57 & \\
\hline
\end{tabular}

Table 11. number of times machines run per day

\begin{tabular}{|l|l|l|}
\hline Number of times machines run per day & \multicolumn{1}{c|}{ frequency } & \multicolumn{1}{c|}{$\%$} \\
\hline 1 & 17 & 39.5 \\
\hline 2 & 21 & 48.8 \\
\hline 3 & 5 & 11.6 \\
\hline Total & 43 & 100.00 \\
\hline
\end{tabular}

Table 12. Average number of samples test per day

\begin{tabular}{|l|l|l|}
\hline Average number of samples test per day & frequency & \multicolumn{1}{c|}{$\%$} \\
\hline $1-4$ & 19 & 44.2 \\
\hline $5-8$ & 16 & 37.2 \\
\hline $9-20$ & 4 & 9.3 \\
\hline $21-30$ & 4 & 9.3 \\
\hline Total & 43 & 100.00 \\
\hline
\end{tabular}

Table 13. Type of problems faced in operating machine (multiple responses)

\begin{tabular}{|l|l|l|}
\hline \multicolumn{1}{|c|}{ Type of problem faced } & frequency & \multicolumn{1}{c|}{$\%$} \\
\hline No problem & 5 & 11.6 \\
\hline Load shading & 5 & 11.6 \\
\hline Voltage up-down & 7 & 16.3 \\
\hline Inadequate modules compared to workload & 4 & 9.3 \\
\hline Module failure & 29 & 67.4 \\
\hline In-adequate cartridge supply & 7 & 16.3 \\
\hline Delay in maintenance & 20 & 46.5 \\
\hline Laptop problem & 2 & 4.7 \\
\hline
\end{tabular}


Table 14. Type of support needed

\begin{tabular}{|l|l|l|}
\hline \multicolumn{1}{|c|}{ Type of support } & \multicolumn{1}{c|}{ frequency } & \multicolumn{1}{c|}{$\%$} \\
\hline No support is needed & 1 & 2.3 \\
\hline More Xpert machine & 3 & 7.3 \\
\hline Refresher training & 40 & 93.0 \\
\hline Software training & 8 & 18.6 \\
\hline Maintenance training & 34 & 79.1 \\
\hline
\end{tabular}

Table 15: Distribution of Respondents (for in-depth interview) by institution

\begin{tabular}{|l|c|}
\hline \multicolumn{1}{|c|}{ Institution/organization } & Number of Respondent \\
\hline Chest Disease Hospital (CDH) & 4 \\
\hline NTP Bangladesh & 4 \\
\hline Damien Foundation Bangladesh & 1 \\
\hline BRAC & 2 \\
\hline Challenge TB (CTB) Bangladesh & 2 \\
\hline TB Hospital & 4 \\
\hline ICDDR,B & 6 \\
\hline Total & 23 \\
\hline
\end{tabular}

Table 16: Distribution of Respondents (for in-depth interview) by their principal responsibility

\begin{tabular}{|l|c|}
\hline principal responsibility & Number of Respondent \\
\hline Managerial & 11 \\
\hline Technical & 7 \\
\hline Supervisory & 5 \\
\hline Total & 23 \\
\hline
\end{tabular}

Table 17 Response from the respondents on these 3 areas (multiple challenges)

\begin{tabular}{|l|c|}
\hline Problem /challenges faced & Frequency \\
\hline Sputum transport mechanism is not well established & 2 \\
\hline Lack of timely maintenance of Machines & 3 \\
\hline Module failure & 9 \\
\hline Frequent error & 3 \\
\hline False result of Rif Resistance due to low bacterial load & 3 \\
\hline Lack of appropriate centrifuge machine for processing of samples of EP cases & 1 \\
\hline Inadequate manpower & \\
\hline Lesson Learned & 4 \\
\hline Availability of GeneXpert is very less in comparison to presumptive cases & 2 \\
\hline Diagnostic algorithm has limited indication for diagnosis of TB and MDR TB & 15 \\
\hline $\begin{array}{l}\text { GeneXpert is a very useful tool for quick and accurate diagnosis of TB and } \\
\text { MDR TB }\end{array}$ & 3 \\
\hline Selection criteria for referring to GeneXpert has limited scope & 1 \\
\hline Incremental yield for Bacteriological cases & 3 \\
\hline $\begin{array}{l}\text { Ambient temp and regular cleanliness (at least once in a month) of filter to keep } \\
\text { the machine well functioning. }\end{array}$ & 2 \\
\hline Well oriented staff can operate the machine nicely & 3 \\
\hline $\begin{array}{l}\text { Error rate is higher in samples of EP TB due to low bacterial load and appropri- } \\
\text { ate centrifuge machine is required for processing }\end{array}$ & 3 \\
\hline
\end{tabular}




\begin{tabular}{|l|c|}
\hline As only one sample is tested, good quality sample is required & 2 \\
\hline $\begin{array}{l}\text { Regular maintenance and calibration should be in place for smooth implemen- } \\
\text { tation of GeneXpert }\end{array}$ & 1 \\
\hline $\begin{array}{l}\text { "Dedicated well trained staff should be there to operate the system for ensuring } \\
\text { quality lab services" }\end{array}$ & 3 \\
\hline $\begin{array}{l}\text { Stable power supply is absolutely necessary. Discontinuation of electricity even } \\
\text { for a fraction of second will cause erroneous test result }\end{array}$ & 1 \\
\hline $\begin{array}{l}\text { "The module replacement process kills lot of time as we have to acquire the } \\
\text { replacement modules from Cepheid, Netherland" }\end{array}$ & 2 \\
\hline Suggestions (if any) for further improvement & 2 \\
\hline A well maintained sputum transportation mechanism to be established & 5 \\
\hline $\begin{array}{l}\text { Diagnostic algorithm needs to be revised to testing all presumptive TB and DR } \\
\text { TB }\end{array}$ & 5 \\
\hline More machines need to made available for easier access & 5 \\
\hline Ensure regular and timely maintenance & 2 \\
\hline $\begin{array}{l}\text { Machine operator needs refresher training including training related to day- to- } \\
\text { day maintenance and software. }\end{array}$ & 2 \\
\hline $\begin{array}{l}\text { Ensure god quality sample in sufficient amount for Xpert testing to produce } \\
\text { accurate results }\end{array}$ & $\begin{array}{l}\text { Diagnostic criteria should be more broadened so that more presumptive TB and } \\
\text { DR TB can be tested. }\end{array}$ \\
\hline
\end{tabular}

\section{References:}

1. Annual TB report 2016, NTP Bangladesh)

2. SAARC Epidemiological response on tuberculosis 2015

3. World Health Organization. Global Tuberculosis Control Report. Geneva, World Health Organization, 2014

4. Global Tuberculosis Report, WHO-2014 \& Global Tuberculosis Report, WHO-2015

5. Zignol M, van Gemert W, Falzon D, et al.Surveillance of anti-tuberculosis drug resistance in the world: an updated analysis, 2007-2010. Bull World Health Organ 2012; 90: 111-119D.

6. Matteelli A, Centis R, D'Ambrosio L, et al.Multidrug-resistant tuberculosis today.Bull World Health Organ 2012; 90: 78.

7. Raviglione $M$, Marais $B$, Floyd $K$,et al. Scaling up interventions to achieve global tuberculosis control: progress and new developments. Lancet 2012; 379: 1902-1913

8. Anonymous2009. Updated guidelines for the use of nucleic acid amplification tests in the diagnosis of tuberculosis. MMWR Morb. Mortal. Wkly. Rep. 58:7-10.
9. Cirillo D. M., et al.2004. Direct rapid diagnosis of rifampicin resistant Mycobacterium tuberculosis infection in clinical samples by line probe assay (INNO LiPA Rif. TB). New Microbiol. 27:221-227.

10. Helb D., et al.2010. Rapid detection of Mycobacterium tuberculosis and rifampin resistance by use of on-demand, near-patient technology. J. Clin. Microbiol. 48:229-237.

11. World Health Organization. Policy Statement: Automated real-time Nucleic Acid Amplification Technology for Rapid and Simultaneous Detection of Tuberculosis and Rifampicin Resistance: Xpert MTB/RIF System. Geneva, World Health Organization, 2011.

12. World Health Organization. Rapid Implementation of the Xpert MTB/RIF Diagnostic Test. Technical and Operational "How-to" Practical Considerations. Geneva, World Health Organization, 2011.

13. Boehme CC, Nicol MP, Nabeta $P$, et al. Feasibility, diagnostic accuracy, and effectiveness of decentralized use of the Xpert MTB/RIF test for diagnosis of tuberculosis and multidrug resistance: a multicentre implementation study.Lancet 2011; 377: 1495-1505 
14. Moure R.,et al.2011. Rapid detection of Mycobacterium tuberculosis complex and rifampin resistance in smear-negative clinical samples by use of an integrated real-time PCR method. J. Clin. Microbiol. 49:1137-1139.

15. Huang W. L., Chen H. Y., Kuo Y. M., Jou R.2009. Performance assessment of the GenoType MTBDRplus test and DNA sequencing in detection of multidrug-resistant Mycobacterium tuberculosis. J. Clin. Microbiol. 47:2520-2524.(13(13)

16. Hillemann $D$., Weizenegger M., Kubica T., Richter E.,Niemann S 2005. Use of the Genotype MTBDR assay for rapid detection of rifampin and isoniazid resistance in Mycobacterium tuberculosis complex isolates. J. Clin. Microbiol. 43:3699-3703

17. Boehme C. C., et al.2010. Rapid molecular detection of tuberculosis and rifampin resistance. N. Engl. J. Med. 363:1005-101

\section{Acknowledgement:}

The technical and financial support was provided by SAARC TB and HIVIIAIDS Centre, Kathmandu, Nepal 